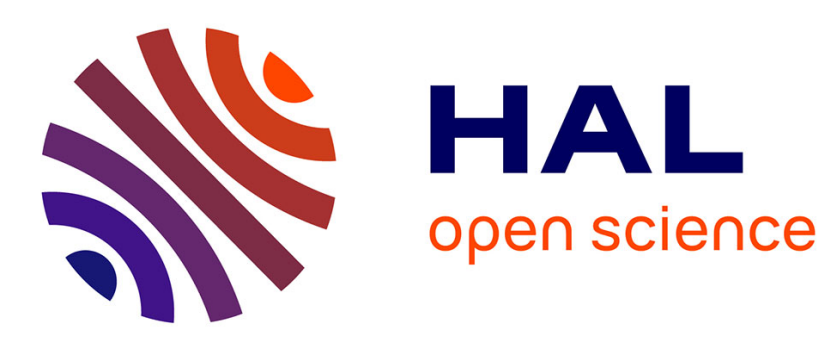

\title{
Yang-Mills and some related algebras
}

Alain Connes, Michel Dubois-Violette

\section{To cite this version:}

Alain Connes, Michel Dubois-Violette. Yang-Mills and some related algebras. Progress in Mathematics, 2007, 251, pp.65-78. hal-00003314v2

\section{HAL Id: hal-00003314 \\ https://hal.science/hal-00003314v2}

Submitted on 19 Nov 2004

HAL is a multi-disciplinary open access archive for the deposit and dissemination of scientific research documents, whether they are published or not. The documents may come from teaching and research institutions in France or abroad, or from public or private research centers.
L'archive ouverte pluridisciplinaire HAL, est destinée au dépôt et à la diffusion de documents scientifiques de niveau recherche, publiés ou non, émanant des établissements d'enseignement et de recherche français ou étrangers, des laboratoires publics ou privés. 


\title{
Yang-Mills and some related algebras
}

\author{
Alain CONNES and Michel DUBOIS-VIOLETTE \\ Collège de France, 3 rue d'Ulm, 75005 Paris, I.H.E.S. and Vanderbilt University, \\ connes@ihes.fr \\ Laboratoire de Physique Théorique, UMR 8627, Université Paris XI, Bâtiment \\ 210, F-91 405 Orsay Cedex, France, Michel.Dubois-Violette@th.u-psud.fr
}

\section{Dedicated to Jacques Bros}

Summary. After a short introduction on the theory of homogeneous algebras we describe the application of this theory to the analysis of the cubic Yang-Mills algebra, the quadratic self-duality algebras, their "super" versions as well as to some generalization.

\section{LPT-ORSAY 04-108}

\subsection{Introduction}

Consider the classical Yang-Mills equations in $(s+1)$-dimensional pseudo Euclidean space $\mathbb{R}^{s+1}$ with pseudo metric denoted by $g_{\mu \nu}$ in the canonical basis of $\mathbb{R}^{s+1}$ corresponding to coordinates $x^{\lambda}$. For the moment the signature plays no role so $g_{\mu \nu}$ is simply a real nondegenerate symmetric matrix with inverse denoted by $g^{\mu \nu}$. In terms of the covariant derivatives $\nabla_{\mu}=\partial_{\mu}+A_{\mu}$ $\left(\partial_{\mu}=\partial / \partial x^{\mu}\right)$ the Yang-Mills equations read

$$
g^{\lambda \mu}\left[\nabla_{\lambda},\left[\nabla_{\mu}, \nabla_{\nu}\right]\right]=0
$$

for $\nu \in\{0, \ldots, s\}$. By forgetting the detailed origin of these equations, it is natural to consider the abstract unital associative algebra $\mathcal{A}$ generated by $(s+1)$ elements $\nabla_{\lambda}$ with the $(s+1)$ cubic relations (1.1). This algebra will be refered to as the Yang-Mills algebra. It is worth noticing here that Equations (1.1) only involve the product through commutators so that, by its very definition the Yang-Mills algebra $\mathcal{A}$ is a universal enveloping algebra.

Our aim here is to present the analysis of the Yang-Mills algebra and of some related algebras based on the recent development of the theory of homogeneous algebras [2], [4]. This analysis is only partly published in [10]. 
In the next section we recall some basic concepts and results on homogeneous algebras which will be used in this paper.

Section 3 is devoted to the Yang-Mills algebra. In this section we recall the definitions and the results of [10]. The proofs are omitted since these are in [10] and since very similar proofs are given in Sections 4 and 6. Instead, we describe the structure of the bimodule resolution of the Yang-Mills algebra and the structure of the corresponding small bicomplexes which compute the Hochschild homology.

In Section 4 we define the super Yang-Mills algebra and we prove for this algebra results which are the counterpart of the results of [10] for the YangMills algebra.

In Section 5 we define and study the super self-duality algebra. In particular, we prove for this algebra the analog of the results of [10] for the self-duality algebra and we point out a very surprising connection between the super selfduality algebra and the algebras occurring in our analysis of noncommutative 3-spheres [9], 11].

In Section 6 we describe some deformations of the Yang-Mills algebra and of the super Yang-Mills algebra.

\subsection{Homogeneous algebras}

Although we shall be concerned in the following with the cubic Yang-Mills algebra $\mathcal{A}$, the quadratic self-duality algebra $\mathcal{A}^{(+)}$[10] and some related algebras, we recall in this section some constructions and some results for general $N$-homogeneous algebras [4], [2]. All vector spaces are over a fixed commutative field $\mathbb{K}$.

A homogeneous algebra of degree $N$ or $N$-homogeneous algebra is an algebra of the form

$$
\mathcal{A}=A(E, R)=T(E) /(R)
$$

where $E$ is a finite-dimensional vector space, $R$ is a linear subspace of $E^{\otimes^{N}}$ and where $(R)$ denotes the two-sided ideal of the tensor algebra $T(E)$ of $E$ generated by $R$. The algebra $\mathcal{A}$ is naturally a connected graded algebra with graduation induced by the one of $T(E)$. To $\mathcal{A}$ is associated another $N$ homogeneous algebra, its dual $\mathcal{A}^{!}=A\left(E^{*}, R^{\perp}\right)$ with $E^{*}$ denoting the dual vector space of $E$ and $R^{\perp} \subset E^{\otimes^{N} *}=E^{* \otimes^{N}}$ being the annihilator of $R$, 迎. The $N$-complex $K(\mathcal{A})$ of left $\mathcal{A}$-modules is then defined to be

$$
\cdots \stackrel{d}{\rightarrow} \mathcal{A} \otimes \mathcal{A}_{n+1}^{! *} \stackrel{d}{\rightarrow} \mathcal{A} \otimes \mathcal{A}_{n}^{! *} \stackrel{d}{\rightarrow} \cdots \stackrel{d}{\rightarrow} \mathcal{A} \rightarrow 0
$$


where $\mathcal{A}_{n}^{! *}$ is the dual vector space of the finite-dimensional vector space $\mathcal{A}_{n}^{!}$of the elements of degree $n$ of $\mathcal{A}^{!}$and where $d: \mathcal{A} \otimes \mathcal{A}_{n+1}^{! *} \rightarrow \mathcal{A} \otimes \mathcal{A}_{n}^{! *}$ is induced by the map $a \otimes\left(e_{1} \otimes \cdots \otimes e_{n+1}\right) \mapsto a e_{1} \otimes\left(e_{2} \otimes \cdots \otimes e_{n+1}\right)$ of $\mathcal{A} \otimes E^{\otimes^{n+1}}$ into $\mathcal{A} \otimes E^{\otimes^{n}}$, remembering that $\mathcal{A}_{n}^{! *} \subset E^{\otimes^{n}}$, (see $\llbracket$ (1]). In (1.2) the factors $\mathcal{A}$ are considered as left $\mathcal{A}$-modules. By considering $\mathcal{A}$ as right $\mathcal{A}$-module and by exchanging the factors one obtains the $N$-complex $\tilde{K}(\mathcal{A})$ of right $\mathcal{A}$-modules

$$
\cdots \stackrel{\tilde{d}}{\rightarrow} \mathcal{A}_{n+1}^{! *} \otimes \mathcal{A} \stackrel{\tilde{d}}{\rightarrow} \mathcal{A}_{n}^{! *} \otimes \mathcal{A} \stackrel{\tilde{d}}{\rightarrow} \cdots \stackrel{\tilde{d}}{\rightarrow} \mathcal{A} \rightarrow 0
$$

where now $\tilde{d}$ is induced by $\left(e_{1} \otimes \cdots \otimes e_{n+1}\right) \otimes a \mapsto\left(e_{1} \otimes \cdots \otimes e_{n}\right) \otimes e_{n+1} a$. Finally one defines two $N$-differentials $d_{\mathbf{L}}$ and $d_{\mathbf{R}}$ on the sequence of $(\mathcal{A}, \mathcal{A})$ bimodules, i.e. of left $\mathcal{A} \otimes \mathcal{A}^{o p p}$-modules, $\left(\mathcal{A} \otimes \mathcal{A}_{n}^{! *} \otimes \mathcal{A}\right)_{n \geq 0}$ by setting $d_{\mathbf{L}}=$ $d \otimes I_{\mathcal{A}}$ and $d_{\mathbf{R}}=I_{\mathcal{A}} \otimes \tilde{d}$ where $I_{\mathcal{A}}$ is the identity mapping of $\mathcal{A}$ onto itself. For each of these $N$-differentials $d_{\mathbf{L}}$ and $d_{\mathbf{R}}$ the sequences

$$
\ldots \stackrel{d_{\mathbf{L}}, d_{\mathbf{R}}}{\rightarrow} \mathcal{A} \otimes \mathcal{A}_{n+1}^{! *} \otimes \mathcal{A} \stackrel{d_{\mathbf{L}}, d_{\mathbf{R}}}{\rightarrow} \mathcal{A} \otimes \mathcal{A}_{n}^{! *} \otimes \mathcal{A} \stackrel{d_{\mathbf{L}}, d_{\mathbf{R}}}{\rightarrow} \ldots
$$

are $N$-complexes of left $\mathcal{A} \otimes \mathcal{A}^{\text {opp }}$-modules and one has

$$
d_{\mathbf{L}} d_{\mathbf{R}}=d_{\mathbf{R}} d_{\mathbf{L}}
$$

which implies that

$$
d_{\mathbf{L}}^{N}-d_{\mathbf{R}}^{N}=\left(d_{\mathbf{L}}-d_{\mathbf{R}}\right)\left(\sum_{p=0}^{N-1} d_{\mathbf{L}}^{p} d_{\mathbf{R}}^{N-p-1}\right)=\left(\sum_{p=0}^{N-1} d_{\mathbf{L}}^{p} d_{\mathbf{R}}^{N-p-1}\right)\left(d_{\mathbf{L}}-d_{\mathbf{R}}\right)=0
$$

in view of $d_{\mathbf{L}}^{N}=d_{\mathbf{R}}^{N}=0$.

As for any $N$-complex [13] one obtains from $K(\mathcal{A})$ ordinary complexes $C_{p, r}(K(\mathcal{A})$ ), the contractions of $K(\mathcal{A})$, by putting together alternatively $p$ and $N-p$ arrows $d$ of $K(\mathcal{A})$. Explicitely $C_{p, r}(K(\mathcal{A}))$ is given by

$$
\ldots \stackrel{d^{N-p}}{\rightarrow} \mathcal{A} \otimes \mathcal{A}_{N k+r}^{! *} \stackrel{d^{p}}{\rightarrow} \mathcal{A} \otimes \mathcal{A}_{N k-p+r}^{! *} \stackrel{d^{N-p}}{\rightarrow} \mathcal{A} \otimes \mathcal{A}_{N(k-1)+r}^{! *} \stackrel{d^{p}}{\rightarrow} \ldots
$$

for $0 \leq r<p \leq N-1$, 胿. These are here chain complexes of free left $\mathcal{A}$-modules. As shown in 4 the complex $C_{N-1,0}(K(\mathcal{A}))$ coincides with the Koszul complex of [2]; this complex will be denoted by $\mathcal{K}(\mathcal{A}, \mathbb{K})$ in the sequel. That is one has

$$
\mathcal{K}_{2 m}(\mathcal{A}, \mathbb{K})=\mathcal{A} \otimes \mathcal{A}_{N m}^{! *}, \quad \mathcal{K}_{2 m+1}(\mathcal{A}, \mathbb{K})=\mathcal{A} \otimes \mathcal{A}_{N m+1}^{! *}
$$

for $m \geq 0$, and the differential is $d^{N-1}$ on $\mathcal{K}_{2 m}(\mathcal{A}, \mathbb{K})$ and $d$ on $\mathcal{K}_{2 m+1}(\mathcal{A}, \mathbb{K})$. If $\mathcal{K}(\mathcal{A}, \mathbb{K})$ is acyclic in positive degrees then $\mathcal{A}$ will be said to be a Koszul algebra. It was shown in [2] and this was confirmed by the analysis of [4] that this is the right generalization for $N$-homogeneous algebra of the usual notion 
of Koszulity for quadratic algebras [17], [16]. One always has $H_{0}(\mathcal{K}(\mathcal{A}, \mathbb{K})) \simeq \mathbb{K}$ and therefore if $\mathcal{A}$ is Koszul, then one has a free resolution $\mathcal{K}(\mathcal{A}, \mathbb{K}) \rightarrow \mathbb{K} \rightarrow 0$ of the trivial left $\mathcal{A}$-module $\mathbb{K}$, that is the exact sequence

$$
\cdots \stackrel{d^{N-1}}{\rightarrow} \mathcal{A} \otimes \mathcal{A}_{N+1}^{! *} \stackrel{d}{\rightarrow} \mathcal{A} \otimes R \stackrel{d^{N-1}}{\rightarrow} \mathcal{A} \otimes E \stackrel{d}{\rightarrow} \mathcal{A} \stackrel{\varepsilon}{\rightarrow} \mathbb{K} \rightarrow 0
$$

of left $\mathcal{A}$-modules where $\varepsilon$ is the projection on degree zero. This resolution is a minimal projective resolution of $\mathcal{A}$ in the graded category [3].

One defines now the chain complex of free $\mathcal{A} \otimes \mathcal{A}^{o p p}$-modules $\mathcal{K}(\mathcal{A}, \mathcal{A})$ by setting

$$
\mathcal{K}_{2 m}(\mathcal{A}, \mathcal{A})=\mathcal{A} \otimes \mathcal{A}_{N m}^{! *} \otimes \mathcal{A}, \quad \mathcal{K}_{2 m+1}(\mathcal{A}, \mathcal{A})=\mathcal{A} \otimes \mathcal{A}_{N m+1}^{! *} \otimes \mathcal{A}
$$

for $m \in \mathbb{N}$ with differential $\delta^{\prime}$ defined by

$$
\begin{gathered}
\delta^{\prime}=d_{\mathbf{L}}-d_{\mathbf{R}}: \mathcal{K}_{2 m+1}(\mathcal{A}, \mathcal{A}) \rightarrow \mathcal{K}_{2 m}(\mathcal{A}, \mathcal{A}) \\
\delta^{\prime}=\sum_{p=0}^{N-1} d_{\mathbf{L}}^{p} d_{\mathbf{R}}^{N-p-1}: \mathcal{K}_{2(m+1)}(\mathcal{A}, \mathcal{A}) \rightarrow \mathcal{K}_{2 m+1}(\mathcal{A}, \mathcal{A})
\end{gathered}
$$

the property $\delta^{\prime 2}=0$ following from (1.6). This complex is acyclic in positive degrees if and only if $\mathcal{A}$ is Koszul, that is if and only if $\mathcal{K}(\mathcal{A}, \mathbb{K})$ is acyclic in positive degrees, 2] and [4]. One always has the obvious exact sequence

$$
\mathcal{A} \otimes E \otimes \mathcal{A} \stackrel{\delta^{\prime}}{\rightarrow} \mathcal{A} \otimes \mathcal{A} \stackrel{\mu}{\rightarrow} \mathcal{A} \rightarrow 0
$$

of left $\mathcal{A} \otimes \mathcal{A}^{o p p}$-modules where $\mu$ denotes the product of $\mathcal{A}$. It follows that if $\mathcal{A}$ is a Koszul algebra then $\mathcal{K}(\mathcal{A}, \mathcal{A}) \stackrel{\mu}{\rightarrow} \mathcal{A} \rightarrow 0$ is a free resolution of the $\mathcal{A} \otimes \mathcal{A}^{o p p}$-module $\mathcal{A}$ which will be refered to as the Koszul resolution of $\mathcal{A}$. This is a minimal projective resolution of $\mathcal{A} \otimes \mathcal{A}^{o p p}$ in the graded category [3].

Let $\mathcal{A}$ be a Koszul algebra and let $\mathcal{M}$ be a $(\mathcal{A}, \mathcal{A})$-bimodule considered as a right $\mathcal{A} \otimes \mathcal{A}^{o p p}$-module. Then, by interpreting the $\mathcal{M}$-valued Hochschild homology $H(\mathcal{A}, \mathcal{M})$ as $H_{n}(\mathcal{A}, \mathcal{M})=\operatorname{Tor}_{n}^{\mathcal{A} \otimes \mathcal{A}^{\text {opp }}}(\mathcal{M}, \mathcal{A})$ [6], the complex $\mathcal{M} \otimes_{\mathcal{A} \otimes \mathcal{A}^{\text {opp }}} \mathcal{K}(\mathcal{A}, \mathcal{A})$ computes the $\mathcal{M}$-valued Hochschild homology of $\mathcal{A}$, (i.e. its homology is the ordinary $\mathcal{M}$-valued Hochschild homology of $\mathcal{A}$ ). We shall refer to this complex as the small Hochschild complex of $\mathcal{A}$ with coefficients in $\mathcal{M}$ and denote it by $\mathcal{S}(\mathcal{A}, \mathcal{M})$. It reads

$$
\ldots \stackrel{\delta}{\rightarrow} \mathcal{M} \otimes \mathcal{A}_{N(m+1)}^{! *} \stackrel{\delta}{\rightarrow} \mathcal{M} \otimes \mathcal{A}_{N m+1}^{! *} \stackrel{\delta}{\rightarrow} \mathcal{M} \otimes \mathcal{A}_{N m}^{! *} \stackrel{\delta}{\rightarrow} \ldots
$$

where $\delta$ is obtained from $\delta^{\prime}$ by applying the factors $d_{L}$ to the right of $\mathcal{M}$ and the factors $d_{R}$ to the left of $\mathcal{M}$. 
Assume that $\mathcal{A}$ is a Koszul algebra of finite global dimension $D$. Then the Koszul resolution of $\mathbb{K}$ has length $D$, i.e. $D$ is the largest integer such that $\mathcal{K}_{D}(\mathcal{A}, \mathbb{K}) \neq 0$. By construction, $D$ is also the greatest integer such that $\mathcal{K}_{D}(\mathcal{A}, \mathcal{A}) \neq 0$ so the free $\mathcal{A} \otimes \mathcal{A}^{\text {opp }}$-module resolution of $\mathcal{A}$ has also length $D$. Thus for a Koszul algebra, the global dimension is equal to the Hochschild dimension. Applying then the functor $\operatorname{Hom}_{\mathcal{A}}(\bullet, \mathcal{A})$ to $\mathcal{K}(\mathcal{A}, \mathbb{K})$ one obtains the cochain complex $\mathcal{L}(\mathcal{A}, \mathbb{K})$ of free right $\mathcal{A}$-modules

$$
0 \rightarrow \mathcal{L}^{0}(\mathcal{A}, \mathbb{K}) \rightarrow \cdots \rightarrow \mathcal{L}^{D}(\mathcal{A}, \mathbb{K}) \rightarrow 0
$$

where $\mathcal{L}^{n}(\mathcal{A}, \mathbb{K})=\operatorname{Hom}_{\mathcal{A}}\left(\mathcal{K}_{n}(\mathcal{A}, \mathbb{K}), \mathcal{A}\right)$. The Koszul algebra $\mathcal{A}$ is Gorenstein iff $H^{n}(\mathcal{L}(\mathcal{A}, \mathbb{K}))=0$ for $n<D$ and $H^{D}(\mathcal{L}(\mathcal{A}, \mathbb{K}))=\mathbb{K}(=$ the trivial right $\mathcal{A}$ module). This is clearly a generalisation of the classical Poincaré duality and this implies a precise form of Poincaré duality between Hochschild homology and Hochschild cohomology [5], [20], 21]. In the case of the Yang-Mills algebra and its deformations which are Koszul Gorenstein cubic algebras of global dimension 3, this Poincaré duality gives isomorphisms

$$
H_{k}(\mathcal{A}, \mathcal{M})=H^{3-k}(\mathcal{A}, \mathcal{M}), \quad k \in\{0,1,2,3\}
$$

between the Hochschild homology and the Hochschild cohomology with coefficients in a bimodule $\mathcal{M}$.

\subsection{The Yang-Mills algebra}

Let $\left(g_{\lambda \mu}\right) \in M_{s+1}(\mathbb{K})$ be an invertible symmetric $(s+1) \times(s+1)$-matrix with inverse $\left(g^{\lambda \mu}\right)$, i.e. $g_{\lambda \mu} g^{\mu \nu}=\delta_{\lambda}^{\nu}$. The Yang-Mills algebra is the cubic algebra $\mathcal{A}$ generated by $s+1$ elements $\nabla_{\lambda}(\lambda \in\{0, \ldots, s\})$ with the $s+1$ relations

$$
g^{\lambda \mu}\left[\nabla_{\lambda},\left[\nabla_{\mu}, \nabla_{\nu}\right]\right]=0, \quad \nu \in\{0, \ldots, s\}
$$

that is $\mathcal{A}=A(E, R)$ with $E=\oplus_{\lambda} \mathbb{K} \nabla_{\lambda}$ and $R \subset E^{\otimes^{3}}$ given by

$$
\begin{aligned}
R & =\sum_{\nu} \mathbb{K} g^{\lambda \mu}\left[\nabla_{\lambda},\left[\nabla_{\mu}, \nabla_{\nu}\right]_{\otimes}\right]_{\otimes} \\
& =\sum_{\rho} \mathbb{K}\left(g^{\rho \lambda} g^{\mu \nu}+g^{\nu \rho} g^{\lambda \mu}-2 g^{\rho \mu} g^{\lambda \nu}\right) \nabla_{\lambda} \otimes \nabla_{\mu} \otimes \nabla_{\nu}
\end{aligned}
$$

In [10] the following theorem was proved.

Theorem 1. The cubic Yang-Mills algebra $\mathcal{A}$ is Koszul of global dimension 3 and is Gorenstein.

The proof of this theorem relies on the computation of the dual cubic algebra $\mathcal{A}^{\text {! }}$ which we now recall. 
The dual $\mathcal{A}^{!}=A\left(E^{*}, R^{\perp}\right)$ of the Yang-Mills algebra is the cubic algebra generated by $s+1$ elements $\theta^{\lambda}(\lambda \in\{0, \ldots, s\})$ with relations

$$
\theta^{\lambda} \theta^{\mu} \theta^{\nu}=\frac{1}{s}\left(g^{\lambda \mu} \theta^{\nu}+g^{\mu \nu} \theta^{\lambda}-2 g^{\lambda \nu} \theta^{\mu}\right) \mathbf{g}
$$

where $\mathbf{g}=g_{\alpha \beta} \theta^{\alpha} \theta^{\beta}$. These relations imply that $\mathbf{g} \in \mathcal{A}_{2}^{!}$is central in $\mathcal{A}^{!}$and that one has $\mathcal{A}_{0}^{!}=\mathbb{K} \mathbb{1} \simeq \mathbb{K}, \mathcal{A}_{1}^{!}=\oplus_{\lambda} \mathbb{K} \theta^{\lambda} \simeq \mathbb{K}^{s+1}, \mathcal{A}_{2}^{!}=\oplus_{\mu \nu} \mathbb{K} \theta^{\mu} \theta^{\nu} \simeq$ $\mathbb{K}^{(s+1)^{2}}, \mathcal{A}_{3}^{!}=\oplus_{\lambda} \mathbb{K} \theta^{\lambda} \mathbf{g} \simeq \mathbb{K}^{s+1}, \mathcal{A}_{4}^{!}=\mathbb{K} \mathbf{g}^{2} \simeq \mathbb{K}$ and $\mathcal{A}_{n}^{!}=0$ for $n \geq 5$. From this, one obtains the description of $[10$ of the Koszul complex $\mathcal{K}(\mathcal{A}, \mathbb{K})$ and the proof of the above theorem. It also follows that the bimodule resolution $\mathcal{K}(\mathcal{A}, \mathcal{A}) \stackrel{\mu}{\rightarrow} \mathcal{A} \rightarrow 0$ of $\mathcal{A}$ reads

$$
0 \rightarrow \mathcal{A} \otimes \mathcal{A} \stackrel{\delta_{3}^{\prime}}{\rightarrow} \mathcal{A} \otimes \mathbb{K}^{s+1} \otimes \mathcal{A} \stackrel{\delta_{2}^{\prime}}{\rightarrow} \mathcal{A} \otimes \mathbb{K}^{s+1} \otimes \mathcal{A} \stackrel{\delta_{1}^{\prime}}{\rightarrow} \mathcal{A} \otimes \mathcal{A} \stackrel{\mu}{\rightarrow} \mathcal{A} \rightarrow 0
$$

where the components $\delta_{k}^{\prime}$ of $\delta^{\prime}$ in the different degrees can be computed by using the description of $\mathcal{K}(\mathcal{A}, \mathbb{K})=C_{2,0}$ given in Section 3 of [10] and are given by

$$
\left\{\begin{array}{l}
\delta_{1}^{\prime}\left(a \otimes e_{\lambda} \otimes b\right)=a \nabla_{\lambda} \otimes b-a \otimes \nabla_{\lambda} b \\
\delta_{2}^{\prime}\left(a \otimes e_{\lambda} \otimes b\right)=\left(g^{\alpha \beta} \delta_{\lambda}^{\gamma}+g^{\beta \gamma} g_{\lambda}^{\alpha}-2 g^{\gamma \alpha} \delta_{\lambda}^{\beta}\right) \times \\
\quad \times\left(a \nabla_{\alpha} \nabla_{\beta} \otimes e_{\gamma} \otimes b+a \nabla_{\alpha} \otimes e_{\gamma} \otimes \nabla_{\beta} b+a \otimes e_{\gamma} \otimes \nabla_{\alpha} \nabla_{\beta} b\right) \\
\quad \delta_{3}^{\prime}(a \otimes b)=g^{\lambda \mu}\left(a \nabla_{\mu} \otimes e_{\lambda} \otimes b-a \otimes e_{\lambda} \otimes \nabla_{\mu} b\right)
\end{array}\right.
$$

where $a, b \in \mathcal{A}, e_{\lambda}(\lambda=0, \ldots, s)$ is the canonical basis of $\mathbb{K}^{s+1}$ and $\nabla_{\lambda}$ are the corresponding generators of $\mathcal{A}$.

Let $\mathcal{M}$ be a bimodule over $\mathcal{A}$. By using the above description of the Koszul resolution of $\mathcal{A}$ one easily obtains the one of the small Hochschild complex $\mathcal{S}(\mathcal{A}, \mathcal{M})$ which reads

$$
0 \rightarrow \mathcal{M} \stackrel{\delta_{3}}{\rightarrow} \mathcal{M} \otimes \mathbb{K}^{s+1} \stackrel{\delta_{2}}{\rightarrow} \mathcal{M} \otimes \mathbb{K}^{s+1} \stackrel{\delta_{1}}{\rightarrow} \mathcal{M} \rightarrow 0
$$

with differential $\delta$ given by

$$
\left\{\begin{array}{l}
\delta_{1}\left(m^{\lambda} \otimes e_{\lambda}\right)=m^{\lambda} \nabla_{\lambda}-\nabla_{\lambda} m^{\lambda}=\left[m^{\lambda}, \nabla_{\lambda}\right] \\
\quad \delta_{2}\left(m^{\lambda} \otimes e_{\lambda}\right)= \\
\quad=\left(\left[\nabla_{\mu},\left[\nabla^{\mu}, m^{\lambda}\right]\right]+\left[\nabla_{\mu},\left[m^{\mu}, \nabla^{\lambda}\right]\right]+\left[m^{\mu},\left[\nabla_{\mu}, \nabla^{\lambda}\right]\right]\right) \otimes e_{\lambda} \\
\delta_{3}(m)=g^{\lambda \mu}\left(m \nabla_{\mu}-\nabla_{\mu} m\right) \otimes e_{\lambda}=\left[m, \nabla^{\lambda}\right] \otimes e_{\lambda}
\end{array}\right.
$$

with obvious notations. By using (1.20) one easily verifies the duality (1.15). For instance $H_{3}(\mathcal{A}, \mathcal{M})$ is $\operatorname{Ker}\left(\delta_{3}\right)$ which is given by the $m \in \mathcal{M}$ such that 
$\nabla_{\lambda} m=m \nabla_{\lambda}$ for $\lambda=0, \ldots, s$ that is such that $a m=m a, \forall a \in \mathcal{A}$, since $\mathcal{A}$ is generated by the $\nabla_{\lambda}$ and it is well known that this coincides with $H^{0}(\mathcal{A}, \mathcal{M})$. Similarily $m^{\lambda} \otimes e_{\lambda}$ is in $\operatorname{Ker}\left(\delta_{2}\right)$ if and only if $\nabla_{\lambda} \mapsto D\left(\nabla_{\lambda}\right)=g_{\lambda \mu} m^{\mu}$ extends as a derivation $D$ of $\mathcal{A}$ into $\mathcal{M}(D \in \operatorname{Der}(\mathcal{A}, \mathcal{M}))$ while $m^{\lambda} \otimes e_{\lambda}=\delta_{3}(m)$ means that this derivation is inner $D=\operatorname{ad}(m) \in \operatorname{Int}(\mathcal{A}, \mathcal{M})$ from which $H_{2}(\mathcal{A}, \mathcal{M})$ identifies with $H^{1}(\mathcal{A}, \mathcal{M})$, and so on.

Assume now that $\mathcal{M}$ is graded in the sense that one has $\mathcal{M}=\oplus_{n \in \mathbb{Z}} \mathcal{M}_{n}$ with $\mathcal{A}_{k} \mathcal{M}_{\ell} \mathcal{A}_{m} \subset \mathcal{M}_{k+\ell+m}$. Then the small Hochschild complex $\mathcal{S}(\mathcal{A}, \mathcal{M})$ splits into subcomplexes $\mathcal{S}(\mathcal{A}, \mathcal{M})=\oplus_{n} \mathcal{S}^{(n)}(\mathcal{A}, \mathcal{M})$ where $\mathcal{S}^{(n)}(\mathcal{A}, \mathcal{M})$ is the subcomplex

$$
0 \rightarrow \mathcal{M}_{n-4} \stackrel{\delta_{3}}{\rightarrow} \mathcal{M}_{n-3} \otimes \mathbb{K}^{s+1} \stackrel{\delta_{2}}{\rightarrow} \mathcal{M}_{n-1} \otimes \mathbb{K}^{s+1} \stackrel{\delta_{1}}{\rightarrow} \mathcal{M}_{n} \rightarrow 0
$$

of (1.19). Assume furthermore that the homogeneous components $\mathcal{M}_{n}$ are finite-dimensional vector spaces, i.e. $\operatorname{dim}\left(\mathcal{M}_{n}\right) \in \mathbb{N}$. Then one has the following Euler-Poincaré formula

$$
\begin{aligned}
& \operatorname{dim}\left(H_{0}^{(n)}\right)-\operatorname{dim}\left(H_{1}^{(n)}\right)+\operatorname{dim}\left(H_{2}^{(n)}\right)-\operatorname{dim}\left(H_{3}^{(n)}\right)= \\
& \operatorname{dim}\left(\mathcal{M}_{n}\right)-(s+1) \operatorname{dim}\left(\mathcal{M}_{n-1}\right)+(s+1) \operatorname{dim}\left(\mathcal{M}_{n-3}\right)-\operatorname{dim}\left(\mathcal{M}_{n-4}\right)
\end{aligned}
$$

for the homology $H^{(n)}$ of the chain complex $\mathcal{S}^{(n)}(\mathcal{A}, \mathcal{M})$.

In the case where $\mathcal{M}=\mathcal{A}$, it follows from the Koszulity of $\mathcal{A}$ that the right hand side of $(1.22)$ vanishes for $n \neq 0$. Denoting as usual by $H H(\mathcal{A})$ the $\mathcal{A}$-valued Hochschild homology of $\mathcal{A}$ which is here the homology of $\mathcal{S}(\mathcal{A}, \mathcal{A})$, we denote by $H H^{(n)}(\mathcal{A})$ the homology of the subcomplex $\mathcal{S}^{(n)}(\mathcal{A}, \mathcal{A})$. Since $\mathcal{A}_{n}=0$ for $n<0$, one has $H H_{0}^{(n)}(\mathcal{A})=0$ for $n<0, H H_{1}^{(n)}(\mathcal{A})=0$ for $n \leq 0$, $H H_{2}^{(n)}(\mathcal{A})=0$ for $n \leq 2$ and $H H_{3}^{(n)}(\mathcal{A})=0$ for $n \leq 3$. Furthermore one has

$$
\begin{gathered}
H H_{0}^{(0)}(\mathcal{A})=H H_{3}^{(4)}(\mathcal{A})=\mathbb{K} \\
H H_{0}^{(1)}(\mathcal{A})=H H_{1}^{(1)}(\mathcal{A})=H H_{2}^{(3)}(\mathcal{A})=\mathbb{K}^{s+1} \\
H H_{0}^{(2)}(\mathcal{A})=H H_{1}^{(2)}(\mathcal{A})=\mathbb{K}^{\frac{(s+1)(s+2)}{2}}
\end{gathered}
$$

and the Euler Poincaré formula reads here

$$
\operatorname{dim}\left(H H_{0}^{(n)}(\mathcal{A})\right)+\operatorname{dim}\left(H H_{2}^{(n)}(\mathcal{A})\right)=\operatorname{dim}\left(H H_{1}^{(n)}(\mathcal{A})\right)+\operatorname{dim}\left(H H_{3}^{(n)}(\mathcal{A})\right)
$$

for $n \geq 1$ which implies

$$
\operatorname{dim}\left(H H_{0}^{(3)}(\mathcal{A})\right)+(s+1)=\operatorname{dim}\left(H H_{1}^{(3)}(\mathcal{A})\right)
$$


for $n=3$ while for $n=1$ and $n=2$ it is already contained in (1.24) and (1.25).

The complete description of the Hochschild homology and of the cyclic homology of the Yang-Mills algebra will be given in [12].

\subsection{The super Yang-Mills algebra}

As pointed out in the introduction, the Yang-Mills algebra is the universal enveloping algebra of a Lie algebra which is graded by giving degree 1 to the generators $\nabla_{\lambda}$ (see in [10]). Replacing the Lie bracket by a super Lie bracket, that is replacing in the Yang-Mills equations (1.1) the commutator by the anticommutator whenever the 2 elements are of odd degrees, one obtains a super version $\tilde{\mathcal{A}}$ of the Yang-Mills algebra $\mathcal{A}$. In other words one defines the super Yang-Mills algebra to be the cubic algebra $\tilde{\mathcal{A}}$ generated $s+1$ elements $S_{\lambda}(\lambda \in\{0, \ldots, s\})$ with the relations

$$
g^{\lambda \mu}\left[S_{\lambda},\left\{S_{\mu}, S_{\nu}\right\}\right]=0, \quad \nu \in\{0, \ldots, s\}
$$

that is $\tilde{\mathcal{A}}=A(\tilde{E}, \tilde{R})$ with $\tilde{E}=\oplus_{\lambda} \mathbb{K} S_{\lambda}$ and $\tilde{R} \subset \tilde{E}^{\otimes^{3}}$ given by

$$
\tilde{R}=\sum_{\rho} \mathbb{K}\left(g^{\rho \lambda} g^{\mu \nu}-g^{\nu \rho} g^{\lambda \mu}\right) S_{\lambda} \otimes S_{\mu} \otimes S_{\nu}
$$

Relations (1.28) can be equivalently written as

$$
\left[g^{\lambda \mu} S_{\lambda} S_{\mu}, S_{\nu}\right]=0, \quad \nu \in\{0, \ldots, s\}
$$

which mean that $g^{\lambda \mu} S_{\lambda} S_{\mu} \in \tilde{\mathcal{A}}_{2}$ is central in $\tilde{\mathcal{A}}$.

It is easy to verify that the dual algebra $\tilde{\mathcal{A}}^{!}=A\left(\tilde{E}^{*}, \tilde{R}^{\perp}\right)$ is the cubic algebra generated by $s+1$ elements $\xi^{\lambda}(\lambda \in\{0, \ldots, s\})$ with the relations

$$
\xi^{\lambda} \xi^{\mu} \xi^{\nu}=-\frac{1}{s}\left(g^{\lambda \mu} \xi^{\nu}-g^{\mu \nu} \xi^{\lambda}\right) \mathbf{g}
$$

where $\mathbf{g}=g_{\alpha \beta} \xi^{\alpha} \xi^{\beta}$. These relations imply that $\mathbf{g} \xi^{\nu}+\xi^{\nu} \mathbf{g}=0$, i.e.

$$
\left\{g_{\lambda \mu} \xi^{\lambda} \xi^{\mu}, \xi^{\nu}\right\}=0, \quad \nu \in\{0, \ldots, s\}
$$

and that one has $\tilde{\mathcal{A}}_{0}^{!}=\mathbb{K} \mathbb{1} \simeq \mathbb{K}, \tilde{\mathcal{A}}_{1}^{!}=\oplus_{\lambda} \mathbb{K} \xi^{\lambda} \simeq \mathbb{K}^{s+1}, \tilde{\mathcal{A}}_{2}^{!}=\oplus_{\mu \nu} \mathbb{K} \xi^{\mu} \xi^{\nu} \simeq$ $\mathbb{K}^{(s+1)^{2}}, \tilde{\mathcal{A}}_{3}^{!}=\oplus_{\lambda} \mathbb{K} \xi^{\lambda} \mathbf{g} \simeq \mathbb{K}^{s+1}, \tilde{\mathcal{A}}_{4}^{!}=\mathbb{K}^{2} \simeq \mathbb{K}$ and $\tilde{\mathcal{A}}_{n}^{!}=0$ for $n \geq 5$.

The Koszul complex $\mathcal{K}(\tilde{\mathcal{A}}, \mathbb{K})$ of $\tilde{\mathcal{A}}$ then reads

$$
0 \rightarrow \tilde{\mathcal{A}} \stackrel{S^{t}}{\rightarrow} \tilde{\mathcal{A}}^{s+1} \stackrel{N}{\rightarrow} \tilde{\mathcal{A}}^{s+1} \stackrel{S}{\rightarrow} \tilde{\mathcal{A}} \rightarrow 0
$$


where $S$ means right multiplication by the column with components $S_{\lambda}, S^{t}$ means right multiplication by the row with components $S_{\lambda}$ and $N$ means right multiplication (matrix product) by the matrix with components

$$
N^{\mu \nu}=\left(g^{\mu \nu} g^{\alpha \beta}-g^{\mu \alpha} g^{\nu \beta}\right) S_{\alpha} S_{\beta}
$$

with $\lambda, \mu, \nu \in\{0, \ldots, s\}$. One has the following result.

Theorem 2. The cubic super Yang-Mills algebra $\tilde{\mathcal{A}}$ is Koszul of global dimension 3 and is Gorenstein.

Proof. By the very definition of $\tilde{\mathcal{A}}$ by generators and relations, the sequence

$$
\tilde{\mathcal{A}}^{s+1} \stackrel{N}{\rightarrow} \tilde{\mathcal{A}}^{s+1} \stackrel{S}{\rightarrow} \tilde{\mathcal{A}} \stackrel{\varepsilon}{\rightarrow} \mathbb{K} \rightarrow 0
$$

is exact. On the other hand it is easy to see that the mapping $\tilde{\mathcal{A}} \stackrel{S^{t}}{\rightarrow} \tilde{\mathcal{A}}^{s+1}$ is injective and that the sequence

$$
0 \rightarrow \tilde{\mathcal{A}} \stackrel{S^{t}}{\rightarrow} \tilde{\mathcal{A}}^{s+1} \stackrel{N}{\rightarrow} \tilde{\mathcal{A}}^{s+1} \stackrel{S}{\rightarrow} \tilde{\mathcal{A}} \stackrel{\varepsilon}{\rightarrow} \mathbb{K} \rightarrow 0
$$

is exact which implies that $\tilde{\mathcal{A}}$ is Koszul of global dimension 3. The Gorenstein property follows from the symmetry by transposition.

The situation is completely similar to the Yang-Mills case, in particular $\tilde{\mathcal{A}}$ has Hochschild dimension 3 and, by applying a result of [15], $\tilde{\mathcal{A}}$ has the same Poincaré series as $\mathcal{A}$ i.e. one has the formula

$$
\sum_{n \in \mathbb{N}} \operatorname{dim}\left(\tilde{\mathcal{A}}_{n}\right) t^{n}=\frac{1}{\left(1-t^{2}\right)\left(1-(s+1) t+t^{2}\right)}
$$

which, as will be shown elsewhere, can be interpreted in terms of the quantum group of the bilinear form $\left(g_{\mu \nu}\right)$ [14] by noting the invariance of Relations (1.30) by this quantum group. For $s=1$ the Yang-Mills algebra and the super Yang-Mills algebra are particular cubic Artin-Schelter algebras [1] whereas for $s \geq 2$ these algebras have exponential growth as follows from Formula (1.32).

\subsection{The super self-duality algebra}

There are natural quotients $\mathcal{B}$ of $\mathcal{A}$ and $\tilde{\mathcal{B}}$ of $\tilde{\mathcal{A}}$ which are connected with parastatistics and which have been investigated in 15. The parafermionic algebra $\mathcal{B}$ is the cubic algebra generated by elements $\nabla_{\lambda}(\lambda \in\{0, \ldots, s\})$ with relations

$$
\left[\nabla_{\lambda},\left[\nabla_{\mu}, \nabla_{\nu}\right]\right]=0
$$

for any $\lambda, \mu, \nu \in\{0, \ldots, s\}$, while the parabosonic algebra $\tilde{\mathcal{B}}$ is the cubic algebra generated by elements $S_{\lambda}(\lambda \in\{0, \ldots, s\})$ with relations 


$$
\left[S_{\lambda},\left\{S_{\mu}, S_{\nu}\right\}\right]=0
$$

for any $\lambda, \mu, \nu \in\{0, \ldots, s\}$. In contrast to the Yang-Mills and the super YangMills algebras $\mathcal{A}$ and $\tilde{\mathcal{A}}$ which have exponential growth whenever $s \geq 2$, these algebras $\mathcal{B}$ and $\tilde{\mathcal{B}}$ have polynomial growth with Poincaré series given by

$$
\sum_{n} \operatorname{dim}\left(\mathcal{B}_{n}\right) t^{n}=\sum_{n} \operatorname{dim}\left(\tilde{\mathcal{B}}_{n}\right) t^{n}=\left(\frac{1}{1-t}\right)^{s+1}\left(\frac{1}{1-t^{2}}\right)^{\frac{s(s+1)}{2}}
$$

but they are not Koszul for $s \geq 2$, [15].

In a sense, the algebra $\mathcal{B}$ can be considered to be somehow trivial from the point of view of the classical Yang-Mills equations in dimension $s+1 \geq 3$ although the algebras $\mathcal{B}$ and $\tilde{\mathcal{B}}$ are quite interesting for other purposes 15 . It turns out that in dimension $s+1=4$ with $g_{\mu \nu}=\delta_{\mu \nu}$ (Euclidean case), the Yang-Mills algebra $\mathcal{A}$ has non trivial quotients $\mathcal{A}^{(+)}$and $\mathcal{A}^{(-)}$which are quadratic algebras refered to as the self-duality algebra and the anti-selfduality algebra respectively $\sqrt{10}$. Let $\varepsilon= \pm$, the algebra $\mathcal{A}^{(\varepsilon)}$ is the quadratic algebra generated by the elements $\nabla_{\lambda}(\lambda \in\{0,1,2,3\}$,$) with relations$

$$
\left[\nabla_{0}, \nabla_{k}\right]=\varepsilon\left[\nabla_{\ell}, \nabla_{m}\right]
$$

for any cyclic permutation $(k, \ell, m)$ of $(1,2,3)$. One passes from $\mathcal{A}^{(-)}$to $\mathcal{A}^{(+)}$by changing the orientation of $\mathbb{K}^{4}$ so one can restrict attention to the self-duality algebra $\mathcal{A}^{(+)}$. This algebra has been studied in [10] where it was shown in particular that it is Koszul of global dimension 2. For further details on this algebra and on the Yang-Mills algebra, we refer to [10] and to the forthcoming paper [12]. Our aim now in this section is to define and study the super version of the self-duality algebra.

Let $\varepsilon=+$ or - and define $\tilde{\mathcal{A}}^{(\varepsilon)}$ to be the quadratic algebra generated by the elements $S_{0}, S_{1}, S_{2}, S_{3}$ with relations

$$
i\left\{S_{0}, S_{k}\right\}=\varepsilon\left[S_{\ell}, S_{m}\right]
$$

for any cyclic permutation $(k, \ell, m)$ of $(1,2,3)$. One has the following.

Lemma 1. Relations (1.33) imply that one has

$$
\left[\sum_{\mu=0}^{3}\left(S_{\mu}\right)^{2}, S_{\lambda}\right]=0
$$

for any $\lambda \in\{0,1,2,3\}$. In other words, $\tilde{\mathcal{A}}^{(+)}$and $\tilde{\mathcal{A}}^{(-)}$are quotients of the super Yang-Mills algebra $\tilde{\mathcal{A}}$ for $s+1=4$ and $g_{\mu \nu}=\delta_{\mu \nu}$.

The proof which is a straightforward verification makes use of the Jacobi identity (see also in 18 ). Thus $\tilde{\mathcal{A}}^{(+)}$and $\tilde{\mathcal{A}}^{(-)}$play the same role with respect 
to $\tilde{\mathcal{A}}$ as $\mathcal{A}^{(+)}$and $\mathcal{A}^{(-)}$with respect to $\mathcal{A}$. Accordingly they will be respectively called the super self-duality algebra and the super anti-self-duality algebra. Again $\tilde{\mathcal{A}}^{(+)}$and $\tilde{\mathcal{A}}^{(-)}$are exchanged by changing the orientation of $\mathbb{K}^{4}$ and we shall restrict attention to the super self-duality algebra in the following, i.e. to the quadratic algebra $\tilde{\mathcal{A}}^{(+)}$generated by $S_{0}, S_{1}, S_{2}, S_{3}$ with relations

$$
i\left\{S_{0}, S_{k}\right\}=\left[S_{\ell}, S_{m}\right]
$$

for any cyclic permutation $(k, \ell, m)$ of $(1,2,3)$. One has the following result.

Theorem 3. The quadratic super self-duality algebra $\tilde{\mathcal{A}}^{(+)}$is a Koszul algebra of global dimension 2.

Proof. One verifies that the dual quadratic algebra $\tilde{\mathcal{A}}^{(+) !}$is generated by elements $\xi^{0}, \xi^{1}, \xi^{2}, \xi^{3}$ with relations $\left(\xi^{\lambda}\right)^{2}=0$, for $\lambda=0,1,2,3$ and $\xi^{\ell} \xi^{m}=$ $-\xi^{m} \xi^{\ell}=i \xi^{0} \xi^{k}=i \xi^{k} \xi^{0}$ for any cyclic permutation $(k, \ell, m)$ of $(1,2,3)$. So one has $\tilde{\mathcal{A}}_{0}^{(+) !}=\mathbb{K} \mathbb{1} \simeq \mathbb{K}, \tilde{\mathcal{A}}_{1}^{(+) !}=\oplus_{\lambda} \mathbb{K} \xi^{\lambda} \simeq \mathbb{K}^{4}, \tilde{\mathcal{A}}_{2}^{(+) !}=\oplus_{k} \mathbb{K} \xi^{0} \xi^{k} \simeq \mathbb{K}^{3}$ and $\tilde{\mathcal{A}}_{n}^{(+) !}=0$ for $n \geq 3$ since the above relations imply $\xi^{\lambda} \xi^{\mu} \xi^{\nu}=0$ for any $\lambda, \mu, \nu \in\{0,1,2,3\}$. The Koszul complex $K\left(\tilde{\mathcal{A}}^{(+)}\right)=\mathcal{K}\left(\tilde{\mathcal{A}}^{(+)}, \mathbb{K}\right)$ (quadratic case) then reads

$$
0 \rightarrow \tilde{\mathcal{A}}^{(+)^{3}} \stackrel{D}{\rightarrow} \tilde{\mathcal{A}}^{(+)^{4}} \stackrel{S}{\rightarrow} \tilde{\mathcal{A}}^{(+)} \rightarrow 0
$$

where $S$ means right matrix product with the column with components $S_{\lambda}$ $(\lambda \in\{0,1,2,3\})$ and $D$ means right matrix product with

$$
D=\left(\begin{array}{cccc}
i S_{1} & i S_{0} & S_{3} & -S_{2} \\
i S_{2} & -S_{3} & i S_{0} & S_{1} \\
i S_{3} & S_{2} & -S_{1} & i S_{0}
\end{array}\right)
$$

It follows from the definition of $\tilde{\mathcal{A}}^{(+)}$by generators and relations that the sequence

$$
\tilde{\mathcal{A}}^{(+)^{3}} \stackrel{D}{\rightarrow} \tilde{\mathcal{A}}^{(+)^{4}} \stackrel{S}{\rightarrow} \tilde{\mathcal{A}}^{(+)} \stackrel{\varepsilon}{\rightarrow} \mathbb{K} \rightarrow 0
$$

is exact. On the other hand one shows easily that the mapping $\tilde{\mathcal{A}}^{(+)^{3}} \stackrel{D}{\rightarrow} \tilde{\mathcal{A}}^{(+)^{4}}$ is injective so finally the sequence

$$
0 \rightarrow \tilde{\mathcal{A}}^{(+) 3} \stackrel{D}{\rightarrow} \tilde{\mathcal{A}}^{(+)^{4}} \stackrel{S}{\rightarrow} \tilde{\mathcal{A}}^{(+)} \stackrel{\varepsilon}{\rightarrow} \mathbb{K} \rightarrow 0
$$

is exact which implies the result.

This theorem implies that the super self-duality algebra $\tilde{\mathcal{A}}^{(+)}$has Hochschild dimension 2 and that its Poincaré series is given by

$$
P_{\tilde{\mathcal{A}}^{(+)}}(t)=\frac{1}{(1-t)(1-3 t)}
$$

in view of the structure of its dual $\tilde{\mathcal{A}}^{(+) !}$described in the proof. Thus everything is similar to the case of the self-duality algebra $\mathcal{A}^{(+)}$. 
Let us recall that the Sklyanin algebra, in the presentation given by Sklyanin [18], is the quadratic algebra $\mathcal{S}\left(\alpha_{1}, \alpha_{2}, \alpha_{3}\right)$ generated by 4 elements $S_{0}, S_{1}, S_{2}, S_{3}$ with relations

$$
\begin{gathered}
i\left\{S_{0}, S_{k}\right\}=\left[S_{\ell}, S_{m}\right] \\
{\left[S_{0}, S_{k}\right]=i \frac{\alpha_{\ell}-\alpha_{m}}{\alpha_{k}}\left\{S_{\ell}, S_{m}\right\}}
\end{gathered}
$$

for any cycic permutation $(k, \ell, m)$ of $(1,2,3)$. One sees that the relations of the super self-duality algebra $\widetilde{\mathcal{A}}^{(+)}$are the relations of the Sklyanin algebra which are independent from the parameters $\alpha_{k}$. Thus one has a sequence of surjective homomorphisms of connected graded algebra

$$
\tilde{\mathcal{A}} \rightarrow \tilde{\mathcal{A}}^{(+)} \rightarrow \mathcal{S}\left(\alpha_{1}, \alpha_{2}, \alpha_{3}\right)
$$

On the other hand for generic values of the parameters the Sklyanin algebra is Koszul Gorenstein of global dimension 4 [19 with the same Poincaré series as the polynomial algebra $\mathbb{K}\left[X_{0}, X_{1}, X_{2}, X_{3}\right]$ and corresponds to the natural ambiant noncommutative 4 -dimensional Euclidean space containing the noncommutative 3-spheres described in [9], [1] (their "homogeneisation"). This gives a very surprising connection between the present study and our noncommutative 3 -spheres for generic values of the parameters. It is worth noticing here that in the analysis of 11] several bridges between noncommutative differential geometry in the sense of [7], [8] and noncommutative algebraic geometry have been established.

\subsection{Deformations}

The aim of this section is to study deformations of the Yang-Mills algebra and of the super Yang-Mills algebra. We use the notations of Sections 3 and 4.

Let the dimension $s+1 \geq 2$ and the pseudo metric $g_{\lambda \mu}$ be fixed and let $\zeta \in P_{1}(\mathbb{K})$ have homogeneous coordinates $\zeta_{0}, \zeta_{1} \in \mathbb{K}$. Define $\mathcal{A}(\zeta)$ to be the cubic algebra generated by $s+1$ elements $\nabla_{\lambda}(\lambda \in\{0, \ldots, s\})$ with relations

$$
\left(\zeta_{1}\left(g^{\rho \lambda} g^{\mu \nu}+g^{\nu \rho} g^{\lambda \mu}\right)-2 \zeta_{0} g^{\rho \mu} g^{\lambda \nu}\right) \nabla_{\lambda} \nabla_{\mu} \nabla_{\nu}=0
$$

for $\rho \in\{0, \ldots, s\}$. The Yang-Mills algebra corresponds to the element $\zeta^{Y M}$ of $P_{1}(\mathbb{K})$ with homogeneous coordinates $\zeta_{0}=\zeta_{1}$. Let $\zeta^{\text {sing }}$ be the element of $P_{1}(\mathbb{K})$ with homogeneous coordinates $\zeta_{0}=\frac{s+2}{2} \zeta_{1}$; one has the following result.

Theorem 4. For $\zeta \neq \zeta^{\text {sing }}$ the cubic algebra $\mathcal{A}(\zeta)$ is Koszul of global dimension 3 and is Gorenstein. 
Proof. The dual algebra $\mathcal{A}(\zeta)^{!}$is the cubic algebra generated by elements $\theta^{\lambda}$ with relations

$$
\theta^{\lambda} \theta^{\mu} \theta^{\nu}=\frac{1}{(s+2) \zeta_{1}-2 \zeta_{0}}\left(\zeta_{1}\left(g^{\lambda \mu} \theta^{\nu}+g^{\mu \nu} \theta^{\lambda}\right)-2 \zeta_{0} g^{\lambda \nu} \theta^{\mu}\right) \mathbf{g}
$$

for $\lambda, \mu, \nu \in\{0, \ldots, s\}$ with $\mathbf{g}=g_{\alpha \beta} \theta^{\alpha} \theta^{\beta}$. This again implies that $\mathbf{g}$ is in the center and that one has $\mathcal{A}_{0}^{!}=\mathbb{K} \mathbb{1} \simeq \mathbb{K}, \mathcal{A}_{1}^{!}=\oplus_{\lambda} \mathbb{K} \theta^{\lambda} \simeq \mathbb{K}^{s+1}, \mathcal{A}_{2}^{!}=$ $\oplus_{\lambda, \mu} \mathbb{K} \theta^{\lambda} \theta^{\mu} \simeq \mathbb{K}^{(s+1)^{2}}, \mathcal{A}_{3}^{!}=\oplus_{\lambda} \mathbb{K} \theta^{\lambda} \mathbf{g} \simeq \mathbb{K}^{s+1}, \mathcal{A}_{4}^{!}=\mathbb{K} \mathbf{g}^{2} \simeq \mathbb{K}$ while $\mathcal{A}_{n}^{!}=0$ for $n \geq 5$, where we have set $\mathcal{A}_{n}^{!}=\mathcal{A}(\zeta)_{n}^{!}$. Setting $\mathcal{A}=\mathcal{A}(\zeta)$, the Koszul complex $\mathcal{K}(\mathcal{A}(\zeta), \mathbb{K})$ of $\mathcal{A}(\zeta)$ reads

$$
0 \rightarrow \mathcal{A} \stackrel{\nabla^{t}}{\rightarrow} \mathcal{A}^{s+1} \stackrel{M}{\rightarrow} \mathcal{A}^{s+1} \stackrel{\nabla}{\rightarrow} \mathcal{A} \rightarrow 0
$$

with the same conventions as before and $M$ with components

$$
M^{\mu \nu}=\frac{1}{(s+2) \zeta_{1}-2 \zeta_{0}}\left(\zeta_{1}\left(g^{\mu \nu} g^{\alpha \beta}+g^{\mu \alpha} g^{\nu \beta}\right)-2 \zeta_{0} g^{\mu \beta} g^{\nu \alpha}\right) \nabla_{\alpha} \nabla_{\beta}
$$

$\mu, \nu, \in\{0, \ldots, s\}$. The theorem follows then by the same arguments as before, using in particular the symmetry by transposition for the Gorenstein property.

It follows that $\mathcal{A}(\zeta)$ has Hochschild dimension 3 and the same Poincaré series as the Yang-Mills algebra for $\zeta \neq \zeta^{\text {sing }}$.

$\underline{\text { Remark. }}$. One can show that the cubic algebra generated by elements $\nabla_{\lambda}$ with relations

$$
\left(\zeta_{1} g^{\rho \lambda} g^{\mu \nu}+\zeta_{2} g^{\nu \rho} g^{\lambda \mu}-2 \zeta_{0} g^{\rho \mu} g^{\lambda \nu}\right) \nabla_{\lambda} \nabla_{\mu} \nabla_{\nu}=0
$$

cannot be Koszul and Gorenstein if $\zeta_{1} \neq \zeta_{2}$ and $\zeta_{0} \neq 0$ or if $\left(\zeta_{1}\right)^{2} \neq\left(\zeta_{2}\right)^{2}$.

Let now $\left(B_{\lambda \mu}\right) \in M_{s+1}(\mathbb{K})$ be an arbitrary invertible $(s+1) \times(s+1)$ matrix with inverse $\left(B^{\lambda \mu}\right)$, i.e. $B_{\lambda \mu} B^{\mu \nu}=\delta_{\lambda}^{\nu}$, and let $\varepsilon=+$ or - . We define $\mathfrak{A}(B, \varepsilon)$ to be the cubic algebra generated by $s+1$ elements $E_{\lambda}$ with relations

$$
\left(B^{\rho \lambda} B^{\mu \nu}+\varepsilon B^{\lambda \mu} B^{\nu \rho}\right) E_{\lambda} E_{\mu} E_{\nu}=0
$$

for $\rho \in\{0, \ldots, s\}$. Notice that $B$ is not assumed to be symmetric. If $B_{\lambda \mu}=g_{\lambda \mu}$ and $\varepsilon=-$ then $\mathfrak{A}(g,-)$ is the super Yang-Mills algebra $\tilde{\mathcal{A}}\left(E_{\lambda} \mapsto S_{\lambda}\right)$ while if $B_{\lambda \mu}=g_{\lambda \mu}$ and $\varepsilon=+$ then $\mathfrak{A}(g,+)$ is $\mathcal{A}\left(\zeta^{0}\right)\left(E_{\lambda} \mapsto \nabla_{\lambda}\right)$ where $\zeta^{0}$ has homogeneous coordinates $\zeta_{1} \neq 0$ and $\zeta_{0}=0$. Thus $\mathfrak{A}(B,+)$ and $\mathfrak{A}(B,-)$ belong to deformations of the Yang-Mills and of the super Yang-Mills algebra respectively.

Theorem 5. Assume that $1+\varepsilon B^{\rho \lambda} B^{\mu \nu} B_{\mu \lambda} B_{\rho \nu} \neq 0$, then $\mathfrak{A}(B, \varepsilon)$ is Koszul of global dimension 3 and is Gorenstein. 
Proof. The Koszul complex $\mathcal{K}(\mathfrak{A}(B, \varepsilon), \mathbb{K})$ can be put in the form

$$
0 \rightarrow \mathfrak{A} \stackrel{E^{t}}{\rightarrow} \mathfrak{A}^{s+1} \stackrel{L}{\rightarrow} \mathfrak{A}^{s+1} \stackrel{E}{\rightarrow} \mathfrak{A} \rightarrow 0
$$

where $\mathfrak{A}=\mathfrak{A}(B, \varepsilon)$ and with the previous conventions, the matrix $L$ being given by

$$
L^{\mu \nu}=\left(B^{\mu \alpha} B^{\beta \nu}+\varepsilon B^{\nu \mu} B^{\alpha \beta}\right) E_{\alpha} E_{\beta}
$$

for $\mu, \nu \in\{0, \ldots, s\}$. The arrow $\mathfrak{A} \stackrel{E^{t}}{\rightarrow} \mathfrak{A}^{s+1}$ is always injective and the exactness of $\mathfrak{A} \stackrel{E^{t}}{\rightarrow} \mathfrak{A}^{s+1} \stackrel{L}{\rightarrow} \mathfrak{A}^{s+1}$ follows from the condition $1+\varepsilon B^{\rho \lambda} B^{\mu \nu} B_{\mu \lambda} B_{\rho \nu} \neq 0$. On the other hand, by definition of $\mathcal{A}$ by generators and relations, the sequence $\mathfrak{A}^{s+1} \stackrel{L}{\rightarrow} \mathfrak{A}^{s+1} \stackrel{E}{\rightarrow} \mathfrak{A} \stackrel{\varepsilon}{\rightarrow} \mathbb{K} \rightarrow 0$ is exact. This shows that $\mathfrak{A}$ is Koszul of global dimension 3. The Gorenstein property follows from (see also in [1])

$$
B^{\rho \lambda} B^{\mu \nu}+\varepsilon B^{\nu \rho} B^{\lambda \mu}=\varepsilon\left(B^{\nu \rho} B^{\lambda \mu}+\varepsilon B^{\mu \nu} B^{\rho \lambda}\right)
$$

for $\rho, \lambda, \mu, \nu \in\{0, \ldots, s\}$.

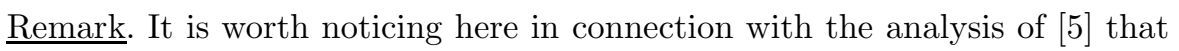
for all the deformations of the Yang-Mills algebra (resp. the super Yang-Mills algebra) considered here which are cubic Koszul Gorenstein algebras of global dimension 3, the dual cubic algebras are Frobenius algebras with structure

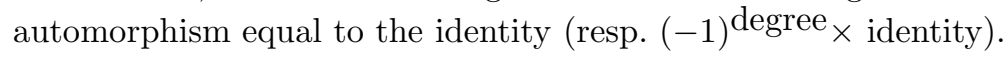

\section{References}

1. M. Artin and W.F. Schelter. Graded algebras of global dimension 3. Adv. Math., 66:171-216, 1987.

2. R. Berger. Koszulity for nonquadratic algebras. J. Algebra, 239:705-734, 2001.

3. R. Berger. La catégorie graduée. 2002.

4. R. Berger, M. Dubois-Violette, and M. Wambst. Homogeneous algebras. J. Algebra, 261:172-185, 2003.

5. R. Berger and N. Marconnet. Koszul and Gorenstein properties for homogeneous algebras. math.QA/0310070, 2003.

6. H. Cartan and S. Eilenberg. Homological algebra. Princeton University Press, 1973.

7. A. Connes. Non-commutative differential geometry. Publ. IHES, 62:257-360, 1986.

8. A. Connes. Non-commutative geometry. Academic Press, 1994.

9. A. Connes and M. Dubois-Violette. Noncommutative finite-dimensional manifolds. I.Spherical manifolds and related examples. Commun. Math. Phys., 230:539-579, 2002.

10. A. Connes and M. Dubois-Violette. Yang-Mills algebra. Lett. Math. Phys., 61:149-158, 2002.

11. A. Connes and M. Dubois-Violette. Moduli space and structure of noncommutative 3-spheres. Lett. Math. Phys., 66:91-121, 2003. 
12. A. Connes and M. Dubois-Violette. Yang-Mills algebra, II. In preparation, 2005.

13. M. Dubois-Violette. $d^{N}=0$ : Generalized homology. K-Theory, 14:371-404, 1998.

14. M. Dubois-Violette and G. Launer. The quantum group of a non-degenerated bilinear form. Phys. Lett., 245B:175-177, 1990.

15. M. Dubois-Violette and T. Popov. Homogeneous algebras, statistics and combinatorics. Lett. Math. Phys., 61:159-170, 2002.

16. J.L. Loday. Notes on Koszul duality for associative algebras. 1999.

17. Yu.I. Manin. Some remarks on Koszul algebras and quantum groups. Ann. Inst. Fourier, Grenoble, 37:191-205, 1987.

18. E.K. Sklyanin. Some algebraic structures connected with the Yang-Baxter equation. Func. Anal. Appl., 16:263-270, 1982.

19. S.P. Smith and J.T. Stafford. Regularity of the four dimensional Sklyanin algebra. Compos. Math., 83:259-289, 1992.

20. M. Van den Bergh. A relation between Hochschild homology and cohomology for Gorenstein rings. Proc. Amer. Math. Soc., 126:1345-1348, 1998.

21. M. Van den Bergh. Erratum. Proc. Amer. Math. Soc., 130:2809-2810, 2002. 